\title{
Integrated Approaches to Analyze Big Data in the Perinatal/Neonatal Space
}

\author{
Rob Knight
}

$\mathbf{T}$ HE HUMAN BODY is made up of more microbial cells than human cells ( $43 \%$ versus $57 \%$ ), and microbial genes outnumber human genes by about 100 to 1 (99\% versus $1 \%$ ). The microbiome plays an essential role in human health and disease and can impact responses to many drugs (e.g., acetaminophen, digoxin, and cyclophosphamide). Many chronic diseases, such as Crohn's disease, multiple sclerosis, type 1 diabetes mellitus, and asthma, have been linked to the microbiome in humans. Furthermore, perturbations to the human microbiome due to overuse of antibiotics may explain the steady increase in chronic diseases seen in industrialized countries. ${ }^{1,2}$

Reduced costs and advances in sequencing technology and applications have allowed researchers to map the microbiome like never before. For example, Quantitative Insights Into Microbial Ecology, or QIIME, is a an open source software package for comparison and analysis of microbial communities. $^{3}$ QIIME can be used to process highthroughput sequencing data into a microbiome map in which all the complexity in a microbial community is distilled down into a single point (Fig. 1). Points closer together are more similar, whereas points further apart are more dissimilar.

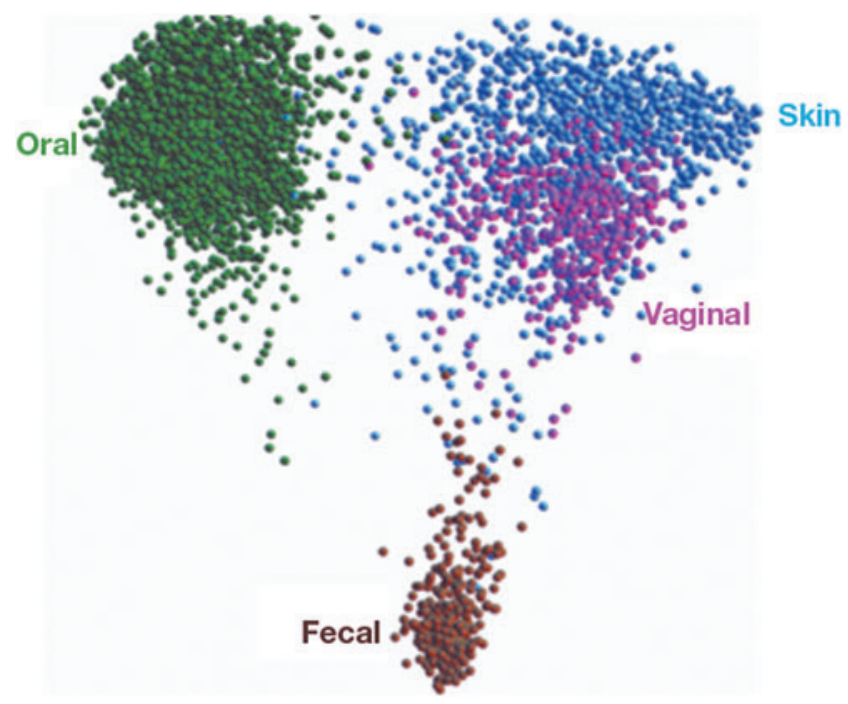

FIG. 1. Map of the human microbiome.
Different parts of the body have completely distinct microbiomes, and these microbiomes can vary based on lifestyle, diet, and environmental factors.

Establishment of the initial microbiome in neonates can vary based on delivery method, with vaginally delivered infants acquiring bacterial communities resembling their mother's vaginal microbiota and infants born by cesarean delivery acquiring bacterial communities similar to those found on their mother's skin (Fig. 2). ${ }^{4}$ Cesarean delivery has been associated with increased risk of immune and metabolic disorders, suggesting initial differences in the microbiome may have important consequences later in life. A small pilot study demonstrated that infants delivered by cesarean section who were exposed to maternal vaginal fluids at birth had microbiota more similar to those of vaginally delivered infants. ${ }^{5}$ Breast milk could also potentially be used to reverse some of the effects of delivery mode, as well as antibiotic use, in infants delivered by cesarean section. ${ }^{6}$ The microbiome

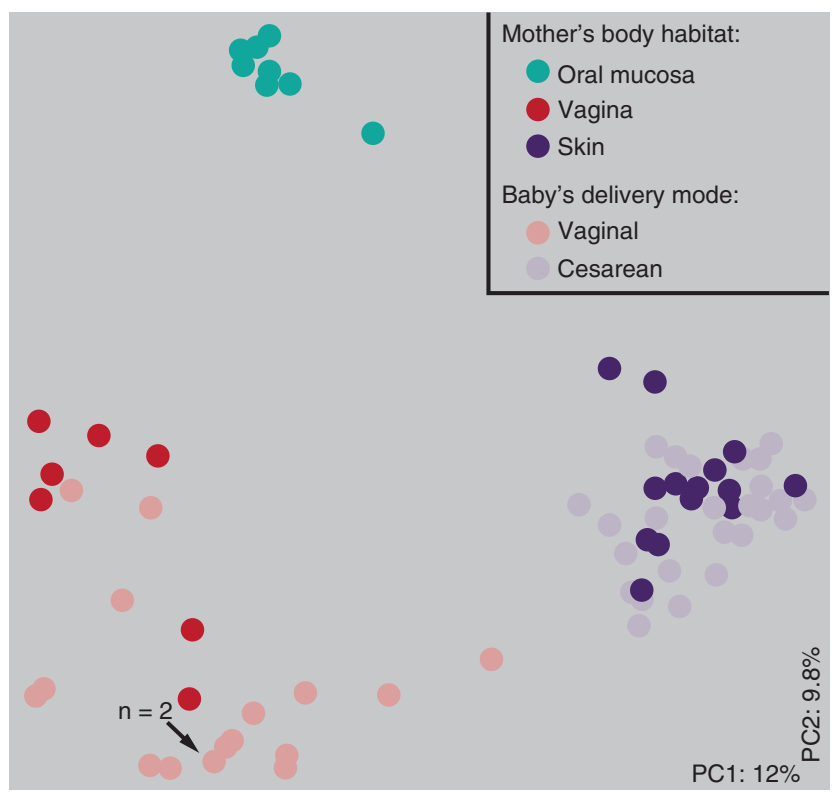

FIG. 2. Delivery mode affects establishment of the initial microbiota in newborns. ${ }^{4}$ 


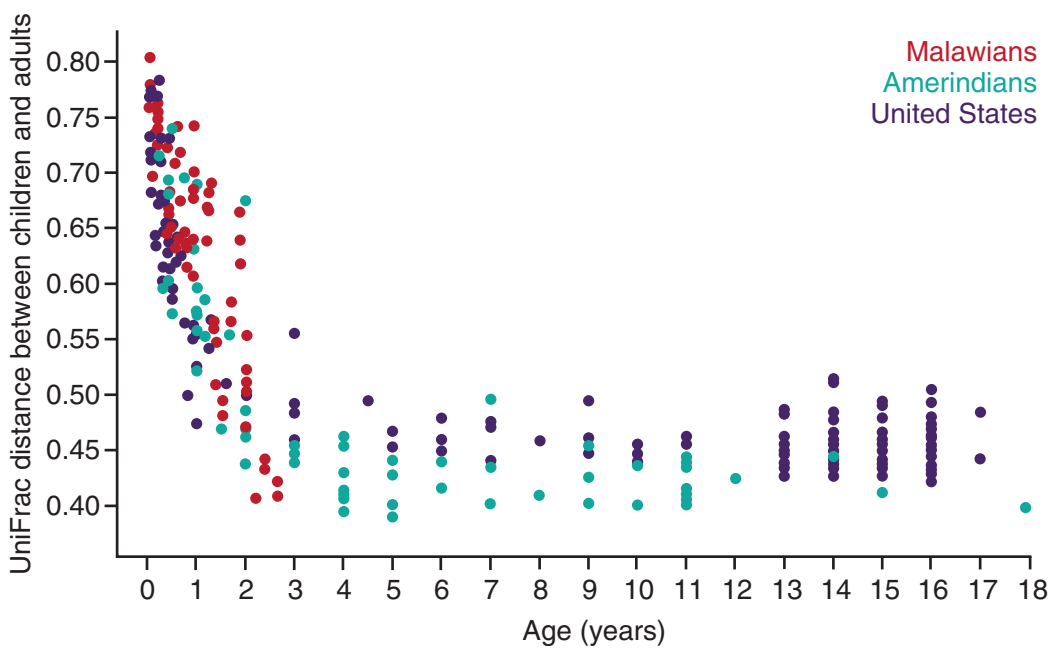

FIG. 3. Differences in fecal microbial communities by age.

continues to evolve during the first few years after birth, reaching an adult-like state by around 3 years of age (Fig. 3). ${ }^{7}$

The American Gut Project is a crowdfunded study designed to compare the microbial diversity of the human gut around the world. Preliminary findings from the study have identified many unanticipated factors that affect the microbiome, such as age, sleep, and the variety of plants consumed. Similar analyses are also being performed in infant populations. Data from the study may also be used to understand the relationship between human diseases and gastrointestinal microbiota. Mapping the microbiome could help determine what interventions (e.g., diet changes and fecal transplants) may help restore a healthy microbiome. This could be an interesting application to help treat necrotizing enterocolitis in preterm infants; changes in the infant microbiome could be tracked through treatment and recovery. Microbial "growth curves" could be developed for other diverse infant populations to measure the impacts of breastfeeding, antibiotic treatment, and delivery mode on the microbiome.

Cystic fibrosis (CF) is an example of a disease in which the microbiome can alter pathogenesis and patient outcomes. Chronic lung infections are a serious and potentially fatal complication of $\mathrm{CF}^{8}$. Studies are underway to generate a three-dimensional map of the microbial chemistry associated with $\mathrm{CF}$ in lungs obtained from end-stage $\mathrm{CF}$ patients requiring lung transplants. ${ }^{9}$ Characterizing the changes in the lung microbiome may help develop more effective and personalized treatments for CF patients. Many of the techniques developed to study the microbiome in $\mathrm{CF}$ and other diseases in adults can also be applied to infant and child populations.

\section{References}

1. Bach JF. The effect of infections on susceptibility to autoimmune and allergic diseases. N Engl J Med 2002;347: 911-920.
2. Blaser MJ. Missing Microbes: How the Overuse of Antibiotics Is Fueling Our Modern Plagues. New York, NY: Henry Holt and Co., 2014.

3. Caporaso JG, Kuczynski J, Stombaugh J, et al. QIIME allows analysis of high-throughput community sequencing data. Nat Methods 2010;7:335-336.

4. Dominguez-Bello MG, Costello EK, Contreras M, et al. Delivery mode shapes the acquisition and structure of the initial microbiota across multiple body habitats in newborns. Proc Natl Acad Sci U S A 2010;107:1197111975.

5. Dominguez-Bello MG, De Jesus-Laboy KM, Shen N, et al. Partial restoration of the microbiota of cesareanborn infants via vaginal microbial transfer. Nat Med 2016; 22:250-253.

6. Bokulich NA, Chung J, Battaglia T, et al. Antibiotics, birth mode, and diet shape microbiome maturation during early life. Sci Transl Med 2016;8:343ra82.

7. Yatsunenko T, Rey FE, Manary MJ, et al. Human gut microbiome viewed across age and geography. Nature 2012;486: 222-227.

8. Cribbs SK, Beck JM. Microbiome in the pathogenesis of cystic fibrosis and lung transplant-related disease. Transl Res 2017; 179:84-96.

9. Garg N, Wang M, Hyde E, et al. Three-dimensional microbiome and metabolome cartography of a diseased human lung. Cell Host Microbe 2017;22:705-716.

Address correspondence to: Rob Knight, PhD University of California San Diego 9500 Gilman Drive, MC 0763 La Jolla, CA 92093

E-mail: robknight@ucsd.edu 